\title{
Reanimation: overcoming objections and obstacles to organ retrieval from non-heart-beating cadaver donors
}

\author{
Robert D Orr, Steven R Gundry and Leonard L Bailey \\ Loma Linda University Medical Center, Loma \\ Linda, California, USA
}

\begin{abstract}
Interest in the retrieval of organs from non-heart-beating cadaver donors has been rekindled by the success of transplantation of solid organs and the insufficient supply of donor organs currently obtained from heart-beating cadaver donors. There are currently two retrieval techniques being evaluated, the in situ cold perfusion approach and the controlled death approach. Both, however, raise ethical concerns. Reanimation is a new method which has been used successfully in animals. We believe this new approach overcomes the ethical objections raised to these other methods.
\end{abstract}

\section{Introduction}

It has previously been assumed that hearts retrieved after anoxic arrest would be poor donor organs because of depleted energy stores and reperfusion necrosis. Prompted by success in using paediatric donor hearts which had prolonged periods of arrest with cardiopulmonary resuscitation efforts for up to two hours, ${ }^{1}$ we have recently reported on successful "reanimation" of dead, pulseless donor hearts in lambs ${ }^{23}$ and baboons. ${ }^{4}$ We believe that such reanimation will be technically feasible in humans and is ethically preferable to currently proposed methods of retrieving hearts from non-heart-beating cadaver donors (NHBCDs).

There are several ethical concerns related to organ retrieval from humans. First is the very definition of death, including the philosophical concept, the legal definition, and the clinical determination of death. There has developed a general consensus and a rather scrupulous following of the unwritten "dead donor rule" in that patients must not be killed by or for organ retrieval. However, what constitutes death varies in different jurisdictions. In some countries, notably Denmark ${ }^{5}$ and Japan, ${ }^{6}$ organ retrieval is not

\section{Key words}

Reanimation; non-heart-beating cadavers; organ retrieval; transplantation. permitted until circulatory death has been deter-을 mined. There has been gradual acceptance by alljurisdictions in the US of the wording of the Uniform Determination of Death Act (UDDA) which says: "An individual who has sustained either:ฏ (1) irreversible cessation of circulatory and respiratory functions; or (2) irreversible cessation of allø functions of the entire brain, including the brain. $v$ stem, is dead. A determination of death must be made in accordance with acceptable medical standards." In the United Kingdom, Pallis ${ }^{8}$ has pro- $\frac{\bar{O}}{0}$ posed that the criterion which best meets the conceptual definition of death of the organism as $a \stackrel{\Phi}{\Phi}$ whole is brain stem death, ie permanent cessation of $\overrightarrow{\vec{F}}$ brain stem functioning only. This concept is used as $\frac{3}{3}$ the basis for diagnosing brain death in the UK. ${ }^{9}$

A second concern is that we should not compro-o mise the care of one living individual for the benefit of another. We do not impose on dying patients who might become organ donors procedures or othero burdens which are counter to their best interests in order to help a potential recipient. Third, it is 0 important to provide humane and dignified care for $₹$ dying patients as well as compassionate care for their음 family members. Fourth, we should be respectful in $>$ our treatment of recently dead bodies.

The primary technical obstacle in organ retrieval, $N$ apart from the conceptual and logistic difficulties of declaring death, has been warm ischaemia time. O This has generated several proposals and mech- $-\omega$ anisms to facilitate organ retrieval. Each should be reviewed with the above ethical concerns in mind.o

In the 1960 s and early 1970 s, organ donors were declared dead by cardiopulmonary criteria before ${ }^{+}$ organs were retrieved. In 1968, because of insufficient donors, a proposal was put forward to declare $\frac{\vec{P}}{\circ}$ death by neurological criteria. ${ }^{10}$ Although there was $\stackrel{?}{\mathscr{Q}}$ some initial opposition to this concept of so-called $\stackrel{\circ}{\mathscr{Q}}$ "brain death", neurological criteria for death gradually became accepted. ${ }^{11}$ There was a rapid transition in most countries to retrieving organs from heart-0 beating cadaver donors (HBCD's). Non-heartbeating cadaver donors were largely abandoned for 
technical, not ethical, reasons. However, the increasing success of transplantation has created a large demand for organs which has not been met by the currently available pool of HBCDs. Attempts have been made to determine the reasons for potential donors failing to become actual donors ${ }^{12}$ and to encourage health professionals to act responsibly in correcting this shortage. ${ }^{13} \mathrm{~A}$ recent proposal to increase the salvage of organs from HBCDs by instituting non-therapeutic ventilation of potential organ donors produced the highest rate of organ donation in the United Kingdom and stabilised the number of patients on dialysis, although the method has faced legal challenge. ${ }^{14}$

Because the number of organs currently being retrieved from $\mathrm{HBCD}$ is still insufficient to meet the need, NHBCDs are once again being considered as a source of transplantable organs. It has been projected that, if implemented on a large scale, this might increase the donor pool by $20-25 \%{ }^{15}$ It is also expected that this move back to NHBCDs might diminish some of the concern expressed by both lay-people and professionals that organs were being removed before "real" death when HBCDs were used. There has already been reported success in the use of kidneys from NHBCDs. ${ }^{16}$

There are two potential sources for NHBCDs. First is the individual who dies a sudden, unexpected death, usually from head trauma or cardiac arrest. Second, there are patients who die a slower and expected death, such as when life support is withdrawn after a decision by patient or surrogate that continued survival is no longer a reasonable or desired goal. In some of the patients in this latter group, death is inevitable and imminent. In others, continued survival is possible but is not desired because of the burdens of continued existence or poor quality of life, for example, a patient who is ventilator-dependent and has minimal awareness of his or her environment.

There have been two proposals for new methods of retrieval of organs from NHBCDs. In situ cold perfusion has been proposed and used by the Regional Organ Bank of Illinois ${ }^{17}$ and controlled death has been proposed and used at the University of Pittsburgh Medical Center. ${ }^{18}$ We offer reanimation as a third option.

\section{In situ cold perfusion}

In situ cold perfusion has been proposed as a way to retrieve solid organs from NHBCDs after sudden, unexpected death, usually in the emergency room. In this proposal, death is declared using standard cardiopulmonary criteria. Intravascular and intraperitoneal perfusion of cold preservation solution is then instituted to reduce the warm ischaemia time. This perfusion may be continued for several hours before organ retrieval is accomplished. It was initially proposed that consent for the retrieval would be obtained before perfusion was begun. This would have met few, if any, ethical objections. However, major logistic problems of obtaining consent within the few minutes available immediately after death made it impossible to retrieve organs in this fashion. An alternative was proposed, namely to begin cold perfusion without consent in order to preserve for the family the option of donation which could then be discussed during the next few hours. ${ }^{19}$

This proposal raises several ethical objections. The objection which is most germane to this clinical situation is the use of a non-therapeutic invasive procedure without consent. Many believe this constitutes disrespectful treatment of a recently dead body. This same objection has been raised in relation to dissection of human corpses by medical students and post-mortem autopsy to determine the cause of death. These procedures have come to be generally acceptable in most cultural settings since it is felt that the potential benefit to others outweighs the potential disrespect to the deceased. Practising endotracheal intubation on recently dead patients, although not disfiguring, continues to be discomfiting to some and an item of ethical discussion. ${ }^{20}$

If in situ cold perfusion is begun prior to obtaining consent, the consent process might still be somewhat hurried for the acutely grieving family, though perhaps no more so than in many instances of retrieval from heart-beating cadaver donors. This proposal also raises the question of whether the family of the deceased will be informed of the cold perfusion if they decline organ donation. The rapid transition from life-saving therapy to organ preservation could entail significant conflict of interest for medical professionals. This obstacle has been adequately addressed in other proposals for organ procurement which clearly delineate and separate the functions of caring for the dying patient and organ retrieval.

It is also uncertain what effect this proposal would have on public acceptance of organ donation. It is not unreasonable to expect, however, that public awareness of cold perfusion without consent could lead to a perception of unethical behavior on the part of the procurement team, and this might decrease the willingness of some families to allow their recently deceased relative to be an organ donor.

\section{Controlled death}

In the Pittsburgh protocol, ${ }^{21}$ the time and place of death is controlled in such a manner as to minimise warm ischaemia and optimise conditions for organ retrieval. This procedure may be requested after a decision has been made by the patient or family to withdraw life-support with the expectation that the patient will die. Under this protocol, unhurried consent may be obtained between the time of the decision to withdraw treatment and the actual 
implementation of that decision. At the appropriate time, the patient is moved to the operating room and prepared for surgery. Life-support is discontinued, with the organ retrieval team waiting in an adjacent room. Death is awaited and declared using new cardiac criteria, and the organs are retrieved.

This proposal was developed methodically over a period of four years with extensive internal and external review. The protocol is quite conservative regarding issues of consent, conflicts of interest, documentation, and review of the decisions. The proposal departs dramatically from medical tradition, however, in its drastic redefinition of death as "two minutes of pulselessness" which may include asystole, ventricular fibrillation, or electro-mechanical disassociation. These new criteria for death have been called, "gerrymandering", 22 "policy creep", ${ }^{23}$ and "an ignoble form of cannibalism." 24 It has been pointed out that the hearts retrieved in this manner do not meet the irreversibility criteria of the UDDA. For instance, these cardiac criteria for death would not be used or acceptable if applied to the potential recipient; heroic efforts would likely be used to resuscitate a patient awaiting an organ if he or she demonstrated ventricular fibrillation. Defenders of the protocol insist that these criteria were developed because of the exceedingly low probability of "auto resuscitation" (ie spontaneous correction of effective pulsation), however, this defence has been criticised because its defenders offer little empiric data to support their contention. ${ }^{25}$

In addition to this major departure from accepted medical standards, this proposal involves the separation of the family from the patient during the dying process and immediately after death. It also raises the difficulty of predicting the time of death so that both family and procurement team may have a protracted wait while separated from the patient and, occasionally patients survive such withdrawal of life-support. Again, the effect on public acceptance of organ donation is unknown, but it is not unreasonable to expect that persons unfamiliar with the details of, or the reasoning behind, the procedure would perceive this hurried retrieval as very discomfiting.

\section{Reanimation}

Reanimation has been performed successfully in five baboons. After being anaesthetised, paralyzed, and extubated, the donor animals demonstrated pulselessness in $7( \pm 1)$ minutes, and asystole followed 9-18 minutes later. The asystolic animals were left undisturbed for 15-31 minutes after which heart retrieval was performed and transplantation was done into recipient baboons. All hearts returned to sinus rhythm without the use of inotropes. Followup showed good myocardial function and no significant ischaemic damage. ${ }^{4}$

Reanimation is proposed by the cardiac transplantation team at Loma Linda University to be used in situations where a decision is made by a patient, or more commonly by a family, to withdraw lifesupport with the expectation that the patient will die. A competent patient might make such a decision when life is being sustained by mechanical ventilation, but he or she finds continued treatment to be too burdensome in spite of maximal efforts to bring comfort, and asks that death-postponing therapy be stopped. Such situations will allow adequate time for unhurried consent from patient or family, as does the Pittsburgh protocol. Under this proposal, however, life-support will be discontinued in the Intensive Care Unit (ICU), and standard cardiac criteria will then be used to declare death. After death is declared, the body could be left undisturbed at room temperature for 20-30 minutes (or longer) Organs could then be retrieved right in the ICU. This proposal would allow the patient's family to remain with the patient during the dying process and for several minutes of personal grieving immediately after death, without being disturbed by the procurement team.

The objection to this proposal could be raised, as in the Pittsburgh protocol, that these donors are not dead because they do not have "irreversible cessation of circulatory and respiratory function" as required by the UDDA. Certainly the reanimated hearts function normally and are therefore not irreversibly dead. The significant difference, however, is that after 20 minutes of asystole, at room temperature, the standard of care for the organism as a whole (versus the heart tissue as a separate entity) is to declare the patient dead by cardiopulmonary criteria without employing resuscitative efforts. In the Guidelines on the Termination of Life-Sustaining Treatment and the Care of the Dying published by the Hastings Center in 1987, it is stated: "Complete cessation of circulation to the normothermic adult brain for more than ten minutes is incompatible with survival of brain tissue". ${ }^{26}$

The criteria for the declaration of death prior to retrieval of hearts in the reanimation procedure is consistent with those proposed by Bernat, Culver and Gert. They observe that the UDDA allows two separate and equal criteria for death. ${ }^{27}$ They contend that conceptually, all death is brain death. They proposed an alternative statute which would read "An individual who has sustained irreversible cessation of all functions of the entire brain, including the brain stem, is dead. (a) In the absence of artificial means of cardiopulmonary support, death may be determined by the prolonged absence of spontaneous circulatory and respiratory functions. (b) In the presence of artificial means of cardiopulmonary support, death must be determined by tests of brain function. In both situations, the determination of death must be made in accordance with accepted medical standards". ${ }^{28}$

Although they believe their proposal has greater conceptual clarity than the UDDA, they feel that the 
advantage of uniformity among states obtained by following the wording of the UDDA outweighs the potential advantage of attempting to change.

\section{Conclusion}

We believe that the proposal for retrieval of solid organs from NHBCDs using this reanimation approach offers several ethical advantages. First, consent is obtained before the protocol is instituted, a major advantage over the in situ cold preservation proposal. Second, using the standard definition of death and accepted standards for declaration of death offers an advantage over the controlled death protocol. We further believe that arranging the retrieval in a manner that will allow the family to be with the patient during and after death is a compassionate measure which will be appreciated by the families and may lessen the potential impact of such a protocol on the general public.

We expect that this method would allow successful solid organ transplantation in adults and children, and by increasing the size of the donor pool will decrease the number of individuals who might otherwise die awaiting the availability of a heart. This proposal has not yet been reviewed by an Institutional Review Board, as is required for research involving human subjects in the US. We offer it here for professional comment.

All three authors work in the Loma Linda University Medical Center of the Loma Linda University School of Medicine. Robert D Orr, MD, is Professor in the School of Medicine's Department of Family Medicine and Director of Clinical Ethics at the University Medical Center. Steven R Gundry, MD, is Professor of Surgery in the School of Medicine's Department of Surgery and Head of the Division of Cardiothoracic Surgery at the University Medical Center. Leonard L Bailey, $M D$, is Professor and Chair of the Department of Surgery in the School of Medicine and Surgeon-in-Chief of the University Medical Center.

\section{References}

1 Kawauchi M, Gundry SR, deBegona AJ, Razzouk AJ, Bailey LL. Utilization of pediatric donors salvaged by cardiopulmonary resuscitation. Fournal of Heart and Lung Transplantation 1993; 12: 185-8.

2 Gundry SR, deBegona AJ, Kawauchi M, Lui H, Razzouk AJ, Bailey LL. Transplantation and reanimation of hearts removed from donors 30 minutes after warm, asystolic "death". Archives of Surgery 1993; 128: 989-93.

3 Gundry SR, deBegona AJ, Kawauchi M, Bailey LL. Successful transplantation of hearts harvested 30 mintues after death from exsanguination. Annals of Thoracic Surgery 1992; 53: 772-5.

4 Gundry SR, Fukashami N, Bailey LL. Successful survival of primates transplanted with "dead", nonheart-beating donors hearts. Fournal of Thoracic and Cardiovascular Surgery 1995; 109: 1097-1102.
5 Danish Council of Ethics. Death criteria: a report. Copenhagen: Danish Council of Ethics, 1988.

6 Kimura R. Japan's dilemma with the definition of death. Kennedy Institute of Ethics fournal 1991; 1: 123-31.

7 Report of Medical Consultants on the Diagnosis of Death to the President's Commission for the Study of $\overline{\bar{\omega}}$ Ethical Problems in Medicine and Biomedical and $\overparen{D}$ Behavioural Research. Guidelines for the determination of death. Fournal of the American Medical Association is 1981; 246: 2184-6.

8 Pallis C. $A B C$ of Brainstem Death. London: British Medical Journal Publishing Group, 1983.

9 Conference of Medical Royal Colleges and their Faculties in the United Kingdom. Diagnosis of brain death. Lancet 1976; 2: 1069-70.

10 Ad Hoc committee of the Harvard Medical School to Examine the Definition of Brain Death. A definition of irreversible coma. Fournal of the American Medical Association 1968; 205: 337-40.

11 President's Commission for the Study of Ethical Problems in Medicine and Biomedical and Behavioural Research. Defining death: medical, legal and ethical issues in the determination of death. Washington, DC: US Government Printing Office, 1981: 1-2, 13-20.

12 Hibbard AD, Pearson IY, McCosker CJ, et al. Potential for cadaveric organ retrieval in New South Wales. British Medical fournal 1992; 304: 1339-43.

13 Mohacsi PJ, Herbertt KL, Thompson JF. Donation and retrieval of cadaveric organs in Australia: accepting the challenge. Medical fournal of Australia 1993; 158: 121-4.

14 Shaw AB. Non-therapeutic (elective) ventilation of potential organ donors: the ethical basis for changing the law. Fournal of Medical Ethics 1996; 22: 72-7.

15 Nathan $\mathrm{H}$. Impact of procuring organs from non-heartbeating cadaver donors. Paper presented at Conference on the Ethical, Psychosocial, and Public Policy Implications of Procuring Organs from Non-HeartBeating Cadaver Donors, 1992 Oct 9-11, Pittsburgh, PA. As cited in Arnold RM, Youngner SJ. Back to the future: obtaining organs from non-heart-beating cadavers. Kennedy Institute of Ethics Fournal 1993; 3: 103-11.

16 Daemen JW, Kootstra G, Wijnen RM, Yin M, Heineman E. Non-heart-beating donors: the Maastricht experience. Clinical Transplantation 1994; 8: 303-16.

17 United Network for Organ Sharing. 1992. UNOS update 8: 15.

18 DeVita MA, Vukmir R, Snyder JV, Graziano C. Procuring organs from a non-heart-beating cadaver: a case report. Kennedy Institute of Ethics fournal 1993; 3: 371-85.

19 DeVita MA, Snyder JV, Grenvik A. History of organ donation by patients with cardiac death. Kennedy Institute of Ethics fournal 1993; 3: 113-29.

20 Orlowski JP, Kanoti GA, Mehlman MJ. The ethical dilemma of permitting teaching and perfecting resuscitation techniques on recently expired patients. fournal of Clinical Ethics 1990; 1: 201-5.

21 University of Pittsburgh Medical Center Policy and Procedure Manual. In Management of terminally ill patients who may become organ donors after death. Kennedy Institute of Ethics fournal 1993; 3: A1-A1 5.

22 Arnold RM, Youngner SJ. The dead donor rule: should we stretch it, bend it, or abandon it? Kennedy Institute of Ethics fournal 1993; 3: 263-78. 
23 Caplan AL. The telltale heart: public policy and the utilization of non-heart-beating donors. Kennedy Institute of Ethics fournal 1993; 3: 251-62.

24 Fox R. "An ignoble form of cannabalism": reflections on the Pittsburgh protocol for procuring organs from non-heart-beating cadavers. Kennedy Institute of Ethics fournal 1993; 3: 231-9.

25 Lynn J. Are the patients who become organ donors under the Pittsburgh protocol for "nonheart-beating donors" really dead? Kennedy
Institute of Ethics fournal 1993; 3: 167-78.

26 Hastings Center. Guidelines on the termination of lifesustaining treatment and the care of the dying. Briarcliff Manor, NY: The Hastings Center, 1987.

27 Bernat J, Culver CM, Gert B. On the definition and criterion of death. Annals of Internal Medicine 1981; 94: 389-94.

28 Bernat J, Culver CM, Gert B. Defining death in theory and practice. Hastings Center Report 1982; 12, 1: 8 .

\section{News and notes}

\section{Lecturer in the Ethics of the New Genetics}

Thanks to a generous donation, the Murdoch Institute for Research into Birth Defects is establishing a post to carry out research into ethics in the context of the new advances in molecular genetics.

The Murdoch Institute is the only Australian Institute of Medical Research with a major interest in human molecular genetics which receives block grant funding from the National Health and Medical Research Council of Australia. It has an active programme of research, including somatic gene therapy, community screening for risk of Down Syndrome and for carrier status for inherited disease, human artificial chromosomes and many other aspects of contemporary genetics. Its associated clinical arm, the Victorian Clinical Genetics Service, offers clinical care on a huband-spoke model for all families with genetic diseases in Victoria and Tasmania, and has done so for thirty years. Its newborn screening programme is regarded as an international model, and it has full diagnostic facilities; it has provided clinical genetic services to over 20,000 families, and offers full training for clinical geneticists, genetic counsellors and molecular geneticists.

The appointment to the post will be at university lecturer level (with the possibility of appointment at senior lecturer grade for an exceptional candidate), for five years (renewable) in the first instance. Full secretarial support, and support for $\mathrm{PhD}$ students/research assistants, will be available subject to the usual academic conditions. There will be occasional teaching duties to medical undergraduates at various points in the medical course, and to genetic counsellors and geneticists, but these will be defined so as to allow the major portion of the appointee's time to be spent carrying out major research programmes in this new area of study. Particularly appropriate areas for research might include ethics of gene therapy, ethics related to genetic screening, autonomy and confidentiality in genetic testing, and the nature of genetic counselling. Applicants would normally be expected to have a postgraduate qualification in ethics and/or medicine and a good research track record. The ability to interact in an open and productive way with colleagues of many different views is essential.

Any person interested in this post should send a full cv, with the names of two academic referees to: Professor Bob Williamson, Director, The Murdoch Institute, PO Box 1100, Parkville 3052, Australia. Fax: 6139348 1391, E-mail: williamb@cryptic.rch.unimelb.edu.au

\section{News and notes}

\section{European Bioethics Seminar}

The sixth European Bioethics Seminar, Health Care Issues in Pluralistic Societies, will be held from August 4-8, 1997, in Nijmegen, the Netherlands. The seminar is organised by the International Program in Bioethics Education and Research. Prominent bioethics scholars from different countries will provide participants with both a formal and practical understanding of contemporary bioethics issues. Special attention will be paid to European traditions in health care ethics. All lectures and plenary sessions will be in English. For more information please contact: Dr B Gordijn, Catholic University of Nijmegen, 232 Dept of Ethics, Philosophy and History of Medicine, PO Box 9101, $6500 \mathrm{HB}$, Nijmegen, the Netherlands. Tel: [31] (0)24-3615320/Fax: [31] (0)24-3540254. 